\title{
Prostate-specific antigen velocity in a prospective prostate cancer screening study of men with genetic predisposition
}

\section{Christos Mikropoulos, Christina G Hutten Selkirk, Sibel Saya, Elizabeth Bancroft, Emily Vertosick, Tokhir} Dadaev, Charles Brendler, Elizabeth Page, Alexander Dias, D Gareth Evans, Jeanette Rothwell, Lovise Maehle, Karol Axcrona, Kate Richardson, Diana Eccles, Thomas Jensen, Palle J Osther, Christi J van Asperen, Hans Vasen, Lambertus A Kiemeney, Janneke Ringelberg, Cezary Cybulski, Dominika Wokolorczyk, Rachel Hart, Wayne Glover, Jimmy Lam, Louise Taylor, Monica Salinas, Lidia Feliubadaló, Rogier Oldenburg, Ruben Cremers, Gerald Verhaegh, Wendy A van Zelst-Stams, Jan C Oosterwijk, Jackie Cook, Derek J Rosario, Saundra S Buys, Tom Conner, Susan Domchek, Jacquelyn Powers, Margreet GEM Ausems, Manuel R Teixeira, Sofia Maia, Louise Izatt, Rita Schmutzler, Kerstin Rhiem, William D Foulkes, Talia Boshari, Rosemarie Davidson, Marielle Ruijs, Apollonia TJM Helderman-van den Enden, Lesley Andrews, Lisa Walker, Katie Snape, Alex Henderson, Irene Jobson, Geoffrey J Lindeman, Annelie Liljegren, Marion Harris, Muriel A Adank, Judy Kirk, Amy Taylor, Rachel Susman, Rakefet Chen-Shtoyerman, Nicholas Pachter, Allan Spigelman, Lucy Side, Janez Zgajnar, Josefina Mora, Carole Brewer, Neus Gadea, Angela F Brady, David Gallagher, Theo van Os, Alan Donaldson, Vigdis Stefansdottir, Julian Barwell, Paul A James, Declan Murphy, Eitan Friedman, Nicola Nicolai, Lynn Greenhalgh, Elias Obeid, Vedang Murthy, Lucia Copakova, John McGrath, Soo-Hwang Teo, Sara Strom, Karin Kast, Daniel A Leongamornlert, Anthony Chamberlain, Jenny Pope, Anna C Newlin, Neil Aaronson, Audrey Ardern-Jones, Chris Bangma, Elena Castro, David Dearnaley, Jorunn Eyfjord, Alison Falconer, Christopher S Foster, Henrik Gronberg, Freddie C Hamdy, Oskar Johannsson, Vincent Khoo, Jan Lubinski, Eli Marie Grindedal, Joanne McKinley, Kylie Shackleton, Anita V Mitra, Clare Moynihan, Gad Rennert, Mohnish Suri, Karen Tricker, The IMPACT study collaborators, Sue Moss, Zsofia Kote-Jarai, Andrew Vickers, Hans Lilja, Brian T Helfand and Rosalind A Eeles

Correction to: British Journal of Cancer (2018) 118, 266-276; doi:10.1038/bjc.2017.429; published online 04 January 2018

The authors would like to acknowledge the following information for this manuscript: Christos Mikropoulos and Christina G Hutten Selkirk are joint first authors of this manuscript. Brian T Helfand and Rosalind A Eeles contributed equally to this manuscript. (c) (1) (2) This work is licensed under the Creative Commons Cay ${ }_{\mathrm{BY}}$ Attribution-Non-Commercial-Share Alike 4.0 International License. To view a copy of this license, visit http:// creativecommons.org/licenses/by-nc-sa/4.0/

(C) The Author(s) named above 2018 